\title{
Amigdalitis de Plaut-Vincent
}

\author{
Plaut-Vincent's tonsillitis \\ Ana Martín-Adrados,* María Lourdes Calleja-Gero, ${ }^{\ddagger}$ Azucena Lloris-Romero-Salazar ${ }^{\S}$ \\ * Residente de Pediatría; ¥ Servicio de Pediatría General; § Servicio de Otorrinolaringología. \\ Hospital Infantil Universitario Niño Jesús, Madrid, España.
}

\section{RESUMEN}

Se presenta el caso de paciente de sexo femenino de 17 años con odinofagia, fiebre y vómitos, a quien en la exploración física se identificó afectación amigdalar unilateral con zonas equimótico-necróticas y vesículas sanguíneas, lo cual fue compatible con diagnóstico de amigdalitis de Plaut-Vincent.

Palabras clave: Adolescente, amigdalitis, amigdalitis de Plaut-Vincent, Fusobacterium necrophorum, Borrelia vincentii.

\section{INTRODUCCIÓN}

La amigdalitis de Plaut-Vincent es una condición aguda de tipo úlcero-necrótico unilateral, causada por una asociación de Fusobacterium necrophorum con Borrelia vincentii. Esta simbiosis, conocida como asociación fusoespirilar, fue descrita por primera vez por Henri Vincent en 1896. Fusobacterium necrophorum es un bacilo Gram negativo, anaerobio estricto, que se encuentra como comensal en la faringe de portadores sanos (7-21\% de la población). Por su parte, Borrelia vincentii es una espiroqueta anaerobia, gramnegativa. ${ }^{1}$

La amigdalitis de Plaut-Vincent predomina en mujeres (2:1), y es más frecuente en pacientes jóvenes con mala higiene dental, fumadores o inmunodeprimidos. ${ }^{2}$ En pacientes con infección por VIH, las lesiones pueden ser muy extensas, dando lugar a un cuadro grave. ${ }^{3}$

\section{ABSTRACT}

We present the case of a 17-year-old female patient with odynophagia, fever and vomiting. On physical examination, unilateral tonsillar involvement was identified with ecchymoticnecrotic areas and blood vesicles, which was compatible with the diagnosis of Plaut-Vincent tonsillitis.

Keywords: Adolescent, tonsillitis, Plaut-Vincent's tonsillitis, Fusobacterium necrophorum, Borrelia vincentii.

Clínicamente suele presentarse con fiebre y odinofagia. En la exploración física se aprecia afectación amigdalar unilateral. Una amígdala estará ulcerada principalmente en el polo superior, cubierta de una membrana necrótica amarillo-grisácea, la cual es friable y se desprende fácilmente dejando un lecho hemorrágico. Puede ocurrir dolor e inflamación de encías, así como existir alguna linfadenopatía homolateral y halitosis. En general, el paciente se encuentra en buen estado general en contraste con la gran afectación local. El diagnóstico es clínico y se puede confirmar mediante estudio microbiológico con la demostración de la asociación fusoespirilar.

Las publicaciones sobre el tema son limitadas, por lo que presentamos el caso de una adolescente con amigdalitis de Plaut-Vincent, con el propósito de que ayude en la detección oportuna de este tipo de pacientes.

Correspondencia: Ana Martín-Adrados, E-mail: martinadradosana@gmail.com

Citar como: Martín-Adrados A, Calleja-Gero ML, Lloris-Romero-Salazar A. Amigdalitis de Plaut-Vincent. Rev Mex Pediatr. 2021; 88(5): 196-198. https://dx.doi.org/10.35366/103900 


\section{PRESENTACIÓN DEL CASO}

Se trata de adolescente de sexo femenino de 17 años, sin antecedentes personales ni familiares de interés. Acude a Urgencias por odinofagia intensa, donde, sin realizarle test rápido de estreptococo, se diagnostica con faringoamigdalitis aguda y se le pauta tratamiento con amoxicilina clavulánico $875 / 125 \mathrm{mg}$ vía oral e ibuprofeno $600 \mathrm{mg}$ cada ocho horas. Tres días después regresa a consulta por persistencia de la odinofagia, fiebre (máxima $38.4{ }^{\circ} \mathrm{C}$ ) y vómitos con restos hemáticos. No refiere disfagia o disnea. A la exploración, la paciente se observa en buen estado general, afebril, con constantes normales (tensión arterial: $97 / 70 \mathrm{mmHg}$, frecuencia cardiaca: $80 \mathrm{lpm}$, saturación basal $\mathrm{O}_{2}: 97 \%$ ), con adecuado estado de nutrición e hidratación, sin exantemas ni petequias.

En cuanto a la exploración de la faringe, se observó la amígdala derecha normal, mientras que la izquierda estaba abombada, hipertrófica y con restos hemáticos en su superficie. No se evidencia trismus, sialorrea, ni alteraciones en úvula o en pilares amigdalinos. Presenta voz algo gangosa. No se detectó alguna adenopatía submandibular. Otoscopía bilateral normal.

Los exámenes de laboratorio mostraron hemograma normal y PCR $2.11 \mathrm{mg} / \mathrm{dL}$. Se toman hemocultivos y exudado faríngeo. Se decide su ingreso para otorgar

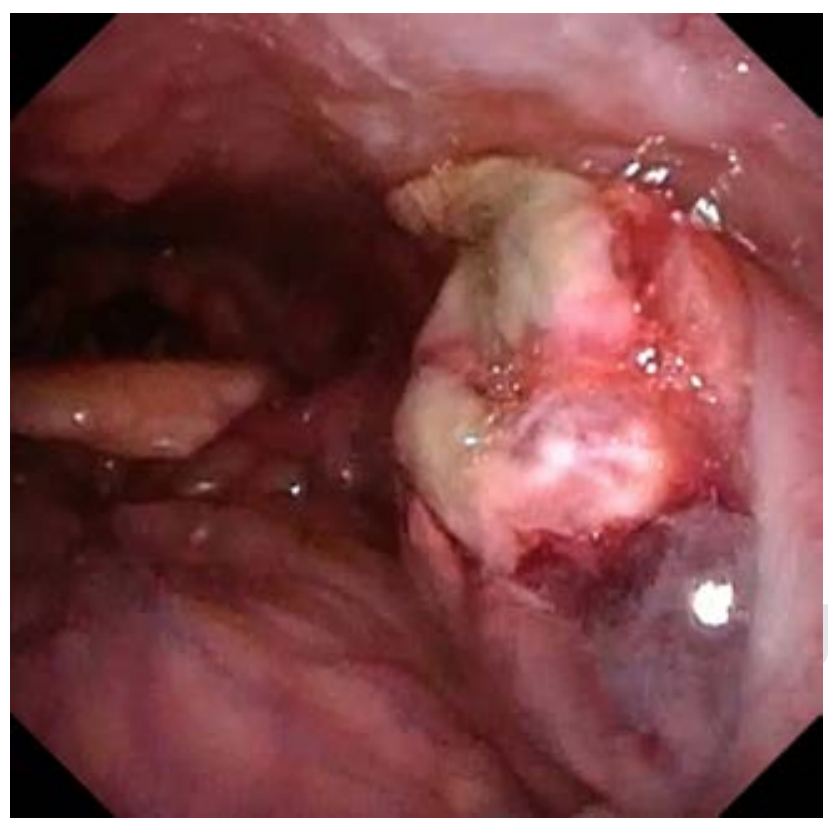

Figura 1: Amígdala izquierda inflamada con zonas equimóticonecróticas y vesículas sanguíneas.

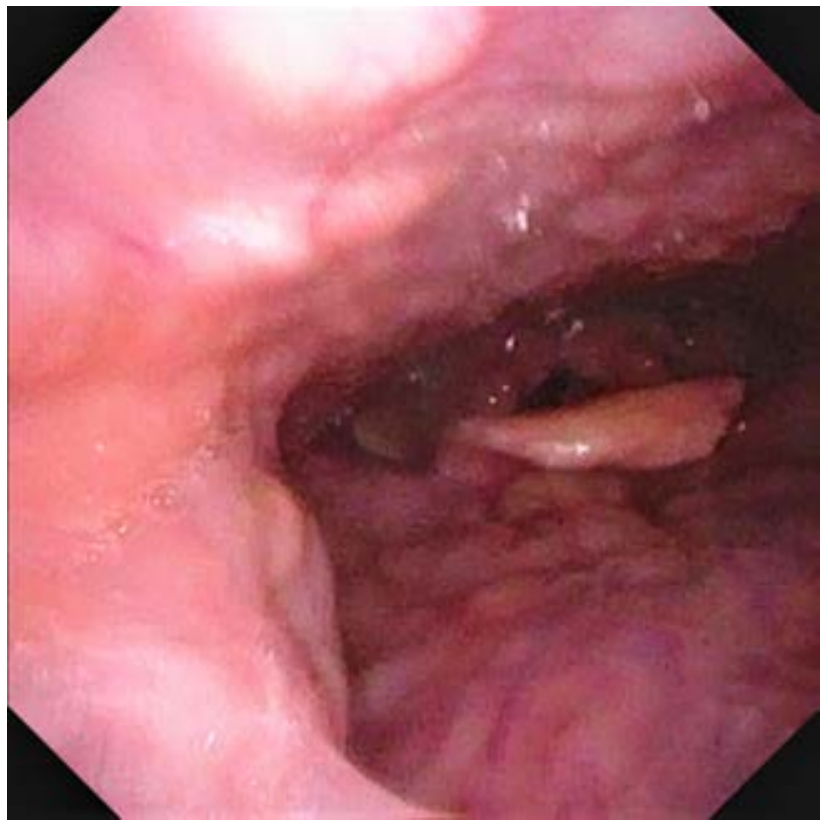

Figura 2: Amígdala derecha normal.

tratamiento antimicrobiano parenteral, ya que se sospechó de absceso periamigdalino.

Se interconsulta al servicio de Otorrinolaringología (ORL), quienes objetivan zonas equimótico-necróticas en la zona superior de amígdala izquierda, que está más protruida sin llegar a desplazar úvula. Por los hallazgos clínicos hacen diagnóstico de amigdalitis de Plaut-Vincent, pero proceden a realizar fibroscopía para confirmar. A través de esta técnica, se visualizó de manera más clara la afectación amigdalina unilateral (Figuras 1 y 2). El hemocultivo fue negativo y en el frotis faríngeo se observaba flora polimicrobiana.

Se administra tratamiento intravenoso (una dosis de corticoide y tres de amoxicilina-clavulánico) con buena evolución, decidiendo su egreso a las 24 horas, pero continuando con amoxicilina-clavulánico 875/125 mg cada 8 horas por vía oral, por 10 días.

\section{DISCUSIÓN}

El diagnóstico de la amigdalitis de Plaut-Vincent es eminentemente clínico y debe ser el primer diagnóstico de sospecha ante una amigdalitis úlcero-necrótica unilateral. ${ }^{4}$ En este caso, inicialmente no se planteó el diagnóstico de angina de Plaut-Vincent, sino el de absceso periamigdalino. El absceso periamigdalino es la complicación más frecuente de una infección amigdalar, la cual consiste en una colección purulenta localizada 
entre la cápsula amigdalar, el músculo constrictor superior de la faringe y el músculo palatofaríngeo. La sintomatología incluye fiebre y odinofagia, y ocasionalmente disfagia o dificultad para movilizar cuello. ${ }^{5}$ En nuestro caso, los expertos de ORL descartan absceso al visualizar las zonas equimótico-necróticas y vesículas sanguíneas amigdalares.

$\mathrm{Al}$ realizar el diagnóstico de amigdalitis de PlautVincent se debe iniciar tratamiento antimicrobiano, pero cuando hay mala evolución se consideran otros diagnósticos, tales como el chancro sifilítico y el carcinoma de amígdala. Cuando la amigdalitis es bilateral, asociada a gingivitis, se debe descartar leucemia o agranulocitosis aguda. ${ }^{6}$

Para esta entidad se recomienda brindar tratamiento antimicrobiano durante 7-10 días; sin embargo, no existe un consenso sobre el tipo específico, ya que para algunos autores es apropiada la penicilina $\mathrm{G}$ o $\mathrm{V},{ }^{6}$ otros sugieren amoxicilina-ácido clavulánico ${ }^{7} \mathrm{o}$ betalactámicos y metronidazol. ${ }^{4}$ Asociado a este tratamiento, se debe ofrecer antiinflamatorio y/o analgésico; además de los cuidados de higiene bucodental tras el episodio agudo. ${ }^{8}$

El tratamiento debe instaurarse rápidamente para prevenir complicaciones, las cuales incluyen el síndrome de Lemierre, tromboflebitis yugular o sepsis postangina. ${ }^{2,9} \mathrm{El}$ síndrome de Lemierre tiene hasta $5 \%$ de mortalidad, su diagnóstico inicial es clínico y se debe sospechar ante una faringoamigdalitis asociada con edema unilateral de cuello. Se confirma con el aislamiento del Fusobacterium necrophorum en hemocultivos para anaerobios. ${ }^{10}$

\section{REFERENCIAS}

1. Cocho Gómez P, Rivero Calle I. Faringoamigdalitis no estreptocócicas ¿hay "algo" más allá de las guías clínicas? Rev Pediatr Aten Primaria. 2015; 17(Suppl 24): 43-52.

2. Omella Ramo S, Torres Santos E, Caballero del Pozo M, Capilla Rodríguez E, Crespo Mahillo M, Paloma Sainz C et al. Amigdalitis unilateral: angina de Plaut-Vincent. Semergen. 2016; 42(Espec Congr 15): 12.

3. Escarrá F, Sormani MI, Litterio M, Isasmendi A, De Bagge M, Parra A. Angina de Vincent Vincent angina. Arch Argent Pediatr. 2019; 117(1): 59-60.

4. De la Fuente Cañibano R, Alañón Fernández MA, Morales Puebla JM. Angina ulceronecrótica unilateral: amigdalitis de Plaut-Vincent. FMC: Form Med Cont Aten Prim. 2016; 23(4): 247-248.

5. Martín Campagne E, del Castillo Martín F, Martínez López MM, Borque de Andrés C, de José Gómez MI, García de Miguel MJ et al. Abscesos periamigdalino y retrofaríngeo, estudio de 13 años. An Esp Pediatr. 2006; 65(1): 32-36.

6. Rodríguez-Rodríguez M, Rodríguez-Rosell V, Rodríguez-Asensio J. Amigdalitis unilateral: angina de Plaut-Vincent. Semergen. 2020; 46(2): e9-e10.

7. Barry B, Bernard S. Infecciones de las vías respiratorias superiores. EMC - Tratado de Medicina. 2018; 22(2): 1-8.

8. Carlson D, Pfadt E. Angina de Vincent y angina de Ludwig: dos infecciones orales peligrosas. Nursing (Ed. española). 2011;29(5): 19-21.

9. Barberán López J, Mensa Pueyo J. Farreras-Rozman. Medicina interna. Cap. 312: Infecciones de la esfera otorrinolaringológica. España: Elsevier; 2020. pp. 2449-2455.

10. Karkos PD, Asrani S, Karkos CD, Leong SC, Theochari EG, Alexopoulou TD et al. Lemierre's syndrome: a systematic review. Laryngoscope. 2009; 119(8): 1552-1559.

Conflicto de intereses: Los autores declaran que no tienen. 